International Journal of Engineering \& Technology, $7(2.7)(2018) 193-196$
International Journal of Engineering \& Technology
SPC
Website: www.sciencepubco.com/index.php/IJET
Research Paper

\title{
Smart garbage collection management system
}

\author{
Mounica Gaddam*1, Venkata Dileep Thatha ${ }^{2}$, Srinivas Ravi Kavuluri ${ }^{3}$, Gopi Krishna Popuri ${ }^{4}$ \\ ${ }^{1}$ B. Tech student, Electronics and Computer Science Engineering, KLEF, Guntur, AP \\ ${ }^{2}$ B. Tech student, Electronics and Computer Science Engineering, KLEF, Guntur, AP \\ ${ }^{3}$ Professor, Electronics and Computer Science Engineering, KLEF, Guntur, AP \\ ${ }^{4}$ Assistant Professor, Electronics and Computer Science Engineering, KLEF, Guntur, AP \\ *E-mail: mounicagaddam22@gmail.com
}

\begin{abstract}
Waste management is necessary in today's world because, with the growing population, waste generated by the human is also increasing. Million Tons of waste is being produced by the people all over the world every day. If waste is not properly disposed off, it may lead to huge health issues and it may have adverse effects on our environment also. Among all, waste collection and transportation are one of the costliest stages in solid waste management. As the truck driver must go to each bin every single day and check whether the bin is full or not. If the bin is not full, it is not only waste of time but also wastes of fuel for truck and it also increases pollution due to smoke released from trucks, needs more men for checking all the bins in different routes. In this paper, we are going to propose a smart solution for this problem using the Internet of Things. We use an ultrasonic sensor to measure the size of the bin, and raspberry pi to process the information further. This sensor data will be sent to the cloud using Wi-Fi module of raspberry pi, from the cloud the data is sent to android app. When the trash inside the bin crosses the certain threshold level, that bin and its location are shown in the App using google maps, and the current location of the truck driver is detected, and shortest path is shown. By this the garbage bins can be emptied before the dustbin overflow.
\end{abstract}

Keywords: Android Ap; Cloud Server; Internet of Things; Shortest Path; Ultrasonic Sensor; Waste Management.

\section{Introduction}

Internet of things is nothing but the devices(things) communicating with each other by using the internet. In internet of things, all the things are connected in a network by using the internet. It is an advanced technology in which all the sensor data is stored in the cloud where data can be easily accessed from the cloud. Sensors and rotors for gathering the data and sending across the internet are also included in this advancement. We use cloud not only to store data but also for data analysis. Such technology can be used in various applications like smart health, smart home, smart agriculture etc., this paper introduces you to the use of internet of things on one such area that is smart City application. In this paper, we are going to implement a smart City application called smart garbage collection management system Where we are going to develop a prototype for collecting the garbage without letting it overflow whenever the garbage fills up to $90 \%$. This project can help in developing the city like Vijayawada into a Smart City.

Vijayawada municipal corporation (VMC) is currently generating about 550 tons of municipal solid waste every day. A total of 33 Dumper beans are being used to transport 350 tons of Municipal solid waste from different places of the city to the nearest station. As per the research of "Central Public Health and environmental engineering organization", 1.3 Pounds of waste is being generated every day by every single person in India. This figure is comparatively less when compared with the waste generated in another state which is approximately 4.6 Pounds every day. But the US population is 307 million in 2009 where the India population is 1.2 billion which is very less when compared to them. According to the above-mentioned statistics, India is generating almost 27 million tons more waste more than the USA every year. The Indian government has already developed some of the methods to manage this garbage in cities. The first method is using an LCD and a load sensor. They have used weight sensor for detecting the weight of the dust inside the bin, however weight sensor will not give any information about the Bin that is empty. The weight sensor will only give the total weight of the bin. So next they have replaced the load sensor with infrared sensor(IR) to sense the distance between the garbage and the dustbin, but this also has some limitations like the IR sensor will Radiate the light and IR rays will be reflected if the waste inside the dustbin is liquid and the IR rays will also not go through the smoke and fog. Due to these limitations it is not very efficient way to know the level of the dust inside the dustbin. Now in this paper, we are replacing the IR sensor with an ultrasonic sensor due to its advantages over IR.

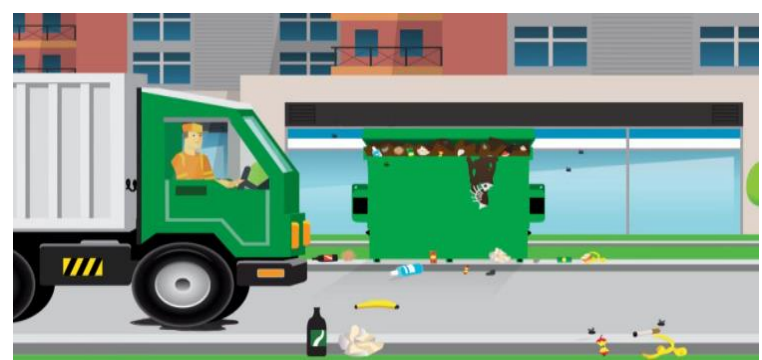

Fig. 1. Dustbin overflowing on streets 
Internet of things can be defined as "A dynamic Global network with self-configuring capabilities based on standards and interoperable Communication protocols where physical and virtual things have identities, physical attributes and virtual personalities use intelligent interfaces and seamlessly integrated into the Information Network." Internet of things applications vary on a large scale. According to IRC IoT applications are classified into smart buildings, smart transportation, Smart energy, smart Technology, smart health as major area. In this paper, the proposed model comes under the Smart City IoT application.

\section{Existing System}

1. It is an integrated system combined with RFID, GPS, GPRS, GIS. In this paper, RFID tags are being mounted on each bin and the trucks will have the RFID reader which will give all the information from the bins when the trucks come closer to the bins GPS will help in tracking the position of the truck and through GPRS the data is being updated from time to time. Drawback: the municipal people will get the information of the bin only when the truck goes near to the bin.

2.It is a combination of ZigBee and Global System for Mobile Communication (GSM). In this paper, they proposed a method such that the sensors present in the bins would sense the level of the bins and give a message to the cleaner using ZIGBEE and GSM technology.

3 In this paper, they have used IR sensor to measure the level of the dustbins in a place and the data is transmitted to the cleaners using GSM technology by using an Arduino. Drawback: In realtime monitoring environment, the IR waves can be blocked by many ways from rain to due, fog and dust particles.

4.In this paper, they have proposed a method for collecting the data related to the dust inside the dustbin and delivery through the wireless mesh network. they have reduced the power consumption by the entire set up by using duty cycle technique. this technique also maximized the operational time.

5. In this paper, they proposed an efficient method with Wireless Sensor Networks (WSN) using VANETs. They will place a laser light diode in the dustbin at a certain height and it will continuously send the laser light towards the photodiode. The concept here is that when the dustbin gets filled with dust the laser light is blocked and it cannot be sensed by the diode when this happens a signal will $\mathrm{b}$ sent to the municipal people that dustbin is filled. Drawback: The light may be absorbed by the liquid is disposed of in the dustbin. From this, it gives the signal to the people without filling it completely.

6. In this paper, they have done a case study on solid waste management in MNIT campus. They have characterized the amount of waste generated and source of waste generation in MNIT Campus They identified the solid waste management practices existing in campus They have suggested some different practices for better management of solid waste management in MNIT Campus.

\section{Proposed System}

\subsection{Ultrasonic Sensor}

Normally, the ultrasonic sensor is used for distance measurement. Here we are using the HC-SR04 ultrasonic module for measuring the length of the bin and amount of garbage inside the bin. It has 4 pins Vcc, GND, trigger, echo. It comprises of a speaker which discharges an ultrasonic sound wave and a mic which recognizes that specific sound wave. Ultrasonic sounds fall under frequency range $100 \mathrm{KH}$ to $50 \mathrm{MH}$, which are above the audible range of frequency. Ultrasonic sensor has transducers which are used for converting one form of energy into another form. Here sound energy is converted into electrical energy. The transmitter will transmit the signal and it waits for some time. If it receives the echo signal the transducer present inside the receiver will convert the echo into an electrical signal. The receiver has piezoelectric material which will vibrate because of echo and this leads to the generation of voltage. The main criteria for setting the time period of triggering pulse is distance.

Initially, we will be placing an ultrasonic sensor to the lid of the dustbin. The ultrasonic sensor placed inside the dustbin will detect the percentage of dust filled by producing sound waves inside the bin. When the trigger pulse is given to the sensor module it sends 8 pulses, frequency of $40 \mathrm{KH}$ ultrasonic sound signals. These signals travel continuously in the air until it hits an object. So, when the signal hits the garbage inside the bin they will reflect. This reflected signal is sensed by the receiver and converted to electrical signals which are given to the raspberry pi board to which the ultrasonic sensor is connected. Considering the time between the transmission and reception of signal we can know the distance by using the following formula.

Distance $=$ Speed $*$ Time $/ 2$

We know that, Speed of sound at sea level $=343 \mathrm{~m} / \mathrm{s}$ or $34300 \mathrm{~cm} / \mathrm{s}$

Distance $=17150 *$ Time

Care should be taken such that, the size of the bin is not too large because the if the object is far away, the noise will be added to the echo signal and the results are manipulated.

\subsection{Raspberry Pi}

We have used Raspberry Pi 3 board for processing the sensor data. Raspberry $\mathrm{Pi}$ is a single board computer having a system on chip Broadcom BCM 2835. It is called mini-computer because of the huge range of features available in it. It has I/O peripherals, GPU, multicore processor, ROM inside and DDR RAM memory, micro HDMI port, USB port, Ethernet port supporting 802.11, Audio and Video jack, camera interface on it. However, the pi board can be powered just by using a 5volts power supply. For this, we can easily connect to a laptop or we can use standard raspberry pi power supply kit.

Raspberry Pi has Linux based operating system. It can support RT Android, Raspbian OS, Windows 10 IoT core, Snappy Ubuntu, RISC OS Pi, RetroPi. Among these, we have used Raspian Jessie. When it comes to coding, if a language can compile on ARM version6 then it can run on Pi board. Scratch, Python, HTML, Javascript, JQuery, Java, C, C++, Perl, are some of the examples Pi can support.Here we have used Python language for coding the Pi.

We can get sensor data locally on a laptop connected to pi using USB before sending the data to the cloud. This can be useful for checking the working of pi and sensors for the designer. With the help of the inbuilt Wi-Fi module in pi, it connects to the thingSpeak cloud platform and pushes the sensor data into the cloud.

\subsection{Cloud Platform}

Here we are using ThingSpeak cloud platform for storing and analysis of the data. This platform is used for storing huge amount of sensor data generated by the devices and sensors connected in the network of the internet of things. It stores data in a wellorganized manner in the form of rows and columns. Using ThingSpeak we can get the percentage of dust filled in each dustbin. It gives the information in the form of graphs. 
Then by using Write API key generated for our channel in Thingspeak, the data generated by the sensors is pushed into the cloud. These API keys are unique for every channel and can be used whenever we want to write data into that corresponding channel. The level of the bin is shown in graphs with the level of dust on $\mathrm{Y}$-axis and time or date on $\mathrm{X}$-axis.

\subsection{Android APP}

In this paper, we have used the android studio for the developing the Android application. Android studio is a programming platform designed to help the programmers for developing and testing Android applications. We know that Thinkspeak consists of sensor data. Using write API key of the channel used in that session we can get the data into the android app. The client needs to set the maximum threshold limit of the dust inside the dustbin. The current garbage level inside the dustbin is displayed in the app. Here we will get the details of the dustbin along with its location $\mathrm{We}$ can get the location of the bin by initially defined unique number to the sensor along with their deployed location. By using google maps API this can be marked as the location of the bin. Based on this data if the level of any bin crosses the pre-defined threshold limit the information regarding the geographical location of the bin will be shown in the android app. If the truck driver selects his current location the app will show the shortest path from his current location to the targeted bin. The app we are designing will detect the current location of the truck driver using GPS in the mobile. Then it will show the shortest path from the current location to the targeted bin using Dijkstra algorithm.

\subsection{Block Diagram}

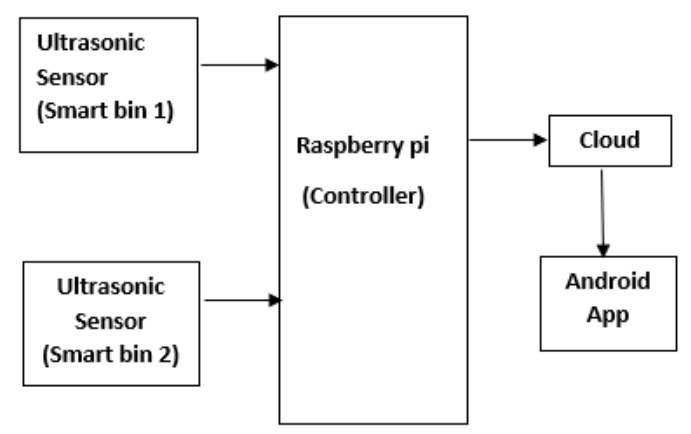

\section{Results}

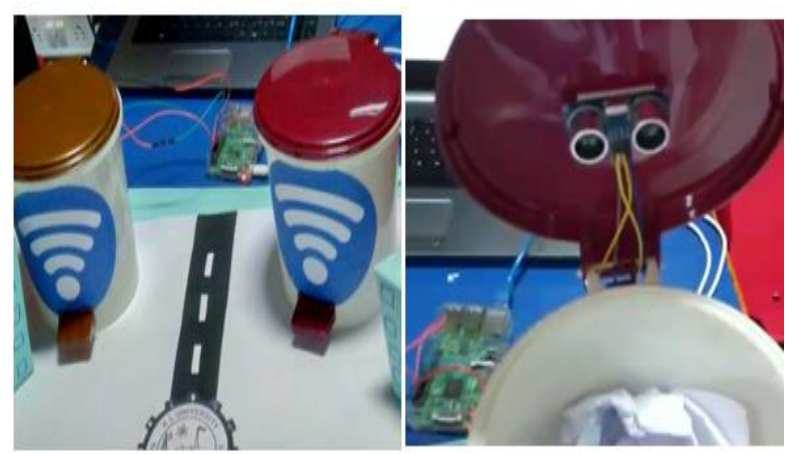

Fig. 2. dustbin with an ultrasonic sensor

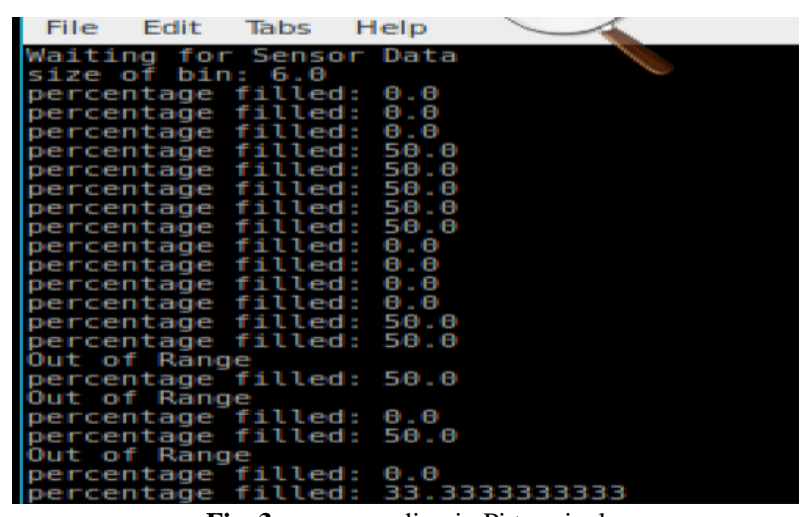

Fig. 3. sensor reading in Pi terminal

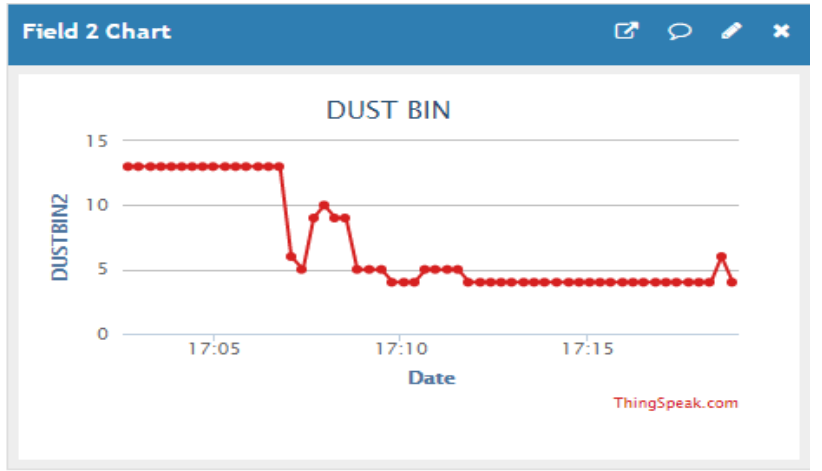

Fig. 4. Sensor data in thingSpeak

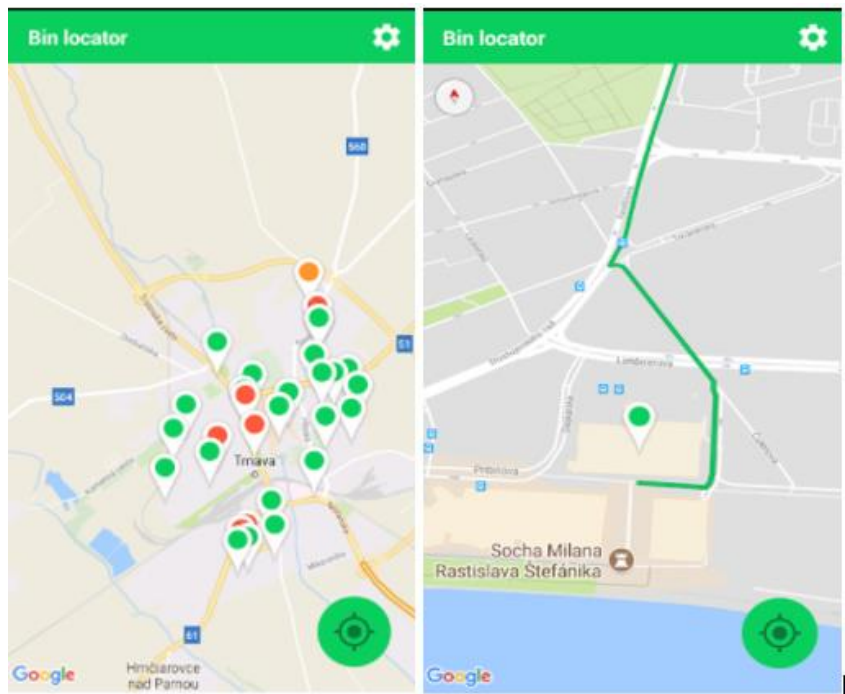

Fig. 5. Android app showing the location of bins and shortest path

\section{Conclusion}

In this paper, we have implemented a system for managing the collection of garbage from the bins by municipal people. This system ensures that the garbage bins are emptied when the garbage inside the bin reached the maximum level. By using this smart approach for collecting the garbage the number of trips for collecting the garbage will decrease since the truck driver need not go every day and check whether the dust bin is full or not. Simply he can go by checking the bin status in the Android app. Predictions can be made based the cloud data such that depending on the area and frequency of dustbin filling up. Using this prediction, the amount of waste generated at regular intervals can be expected and take prior measures before the dustbin gets overflow. By this cost involved in the garbage collection is minimized and no need to employ many truck drivers for checking daily. While comin into environmental advantages, if the trucks go less number of 
rounds in the city the fuel can be saved, and traffic can be minimized to a little extent and the pollution caused by the emission of smoke can be reduced. It ultimately helps in keeping the city clean and can be a part of Swatch Bharath program.

\section{Acknowledgement}

I express my gratitude to Dr. K. Raghav Rao, Head of the Department of Electronics and Computer Science Engineering for providing me with adequate facilities, ways, and means by which I was able to complete this paperwork.

\section{References}

[1] An overview of the solid waste bin monitoring system by Md shafiqul Islam, M.A. Hannam, Maher Arebey from RESEARCH GATE in the year 2012,

[2] Waste Bin Monitoring System Using Integrated Technologies by Kanchan Mahajan1, Prof.J.S. Chitode2 from the international journal of innovative sciences, engineering and technologies, IJIRET in the year 2014

[3] Smart Garbage Management System by Vikrant Bhor1, Pankaj Morajkar2, Amol Deshpande from International Journal of Engineering Research \& Technology (IJERT) in the year 2015

[4] Smart Waste Management System by F chain F olianto, young sheng low, Wai Leong Yeow from IEEETenth International Conference on Intelligent Sensors, Sensor Networks and Information Processing (ISSNIP) in the year 2015

[5] Efficient Garbage Disposal Management in Metropolitan Cities Using VANETs by Narendra Kumar G., Chandrika Swamy, and K. N. Nagadarshini (Journal of Clean Energy Technologies, Vol. 2, No. 3, July 2014)

[6] Solid Waste Collection and Segregation: A Case Study of MIT Campus, Jaipur by Vipin Upadhyay, Jethro A.S, Poonia M. P (International Journal of Engineering and Innovative Technology (IJEIT) Volume 1, Issue 3)

[7] V. Kumar and R.K. Pandit,"Problems of solid waste management in Indian cities", International Journal of Scientific and Research Publications, Volume 3, Issue 3, March 2013.

[8] S. Hu and T. Dai,"Online map application development using Google Maps API, SQL database, and ASP.NET", International Journal of Information and Communication Technology Research.

[9] T. Mohammad,"Using ultrasonic and infrared sensors for distance measurement", International Journal of Mechanical, Aerospace, Industrial, Mechatronic and Manufacturing Engineering Vol:3, No:3, 2009.

[10] Smart Garbage Management System by Vikrant Bhor1, Pankaj Morajkar2, Amol Deshpande from International Journal of Engineering Research \& Technology (IJERT) in the year 2015.

[11] Smartbin: Smart Waste Management System by F chain F olianto, yong sheng low, Wai Leong Yeow from IEEE Tenth International Conference on Intelligent Sensors, Sensor Networks and Information Processing (ISSNIP) in the year 2015 